\title{
SNARE assembly and disassembly exhibit a pronounced hysteresis
}

\author{
Dirk Fasshauer ${ }^{1}$, Wolfram Antonin ${ }^{1}$, Vinod Subramaniam² and Reinhard Jahn ${ }^{1}$
}

Published online: 7 January 2002, DOI: 10.1038/nsb750

SNARE proteins are essential for intracellular membrane fusion of eukaryotes. Their assembly into stable fourhelix bundles bridges membranes and may provide the energy for initiating membrane fusion. In vitro, assembly of soluble SNARE fragments is accompanied by major structural rearrangements that can be described as a folding reaction. The pathw ays and the thermodynamics of SNARE protein interactions, how ever, are not know $\mathrm{n}$. Here we report that assembly and dissociation of two distantly related SNARE complexes exhibit a marked hysteresis. The assembled and disassembled native states are separated by a kinetic barrier and cannot equilibrate on biologically relevant timescales. We suggest that the hysteresis is a hallmark of all SNARE complexes and that complex assembly and disassembly follow different pathw ays that may be independently controlled.

Fusion of lipid bilayers is a fundamental process in eukaryotic cells. During intracellular transport, vesicles bud from one compartment and fuse with their target compartment ${ }^{1}$. Because strong repulsive forces exist between membranes, specialized proteins are required to bring together and fuse the membranes 2,3 . SNARE proteins, which constitute a superfamily of small membrane proteins, aretheleading candidates for mediating intracellular membrane fusion. They assemble into stable heterooligomeric complexes consisting of a central four-helix bundle structure. The complex-forming regions are located adjacent to their respective membrane anchors and consist of homologous heptad repeats of $\sim 60$ residues in length, referred to as SNARE motiff 4,5 .

Although formation of SNARE complexes is believed to be an essential step for fusion, whether SNAREs catalyze membrane fusion directly or whether they are involved in a step that precedes membrane merger is still controversial. In the currently favored model, SNARE proteins from opposing membranes assemble into 'trans'-complexes between membranes, forcing these membranes into close apposition and, thus, overcoming the repulsive forces ${ }^{6}$. After fusion, the chaperone-like ATPase NSF (N-ethylmaleimide-sensitive factor) and its SNAP (soluble NSF attachment protein)-cofactors disassemble the resulting 'cis'-complexes, allowing the SNAREs to be reused.

In support of this model, slow fusion was observed when SNARES (SNAP receptors) were reconstituted into liposomes, suggesting that SNARE assembly between membranes suffices for fusion?. In contrast, studies on homotypic membrane fusion of yeast vacuoles challenge theidea that SNARE proteins operate as fusogen $s^{8}$, suggesting rather that fusion subsequently may be catalyzed by other protein $9^{9,10}$.

To unravel the molecular mechanism by which SNAREs operate in membrane fusion, achieving a detailed picture of the dynamics of and the intermediates in their assembly is essential. a

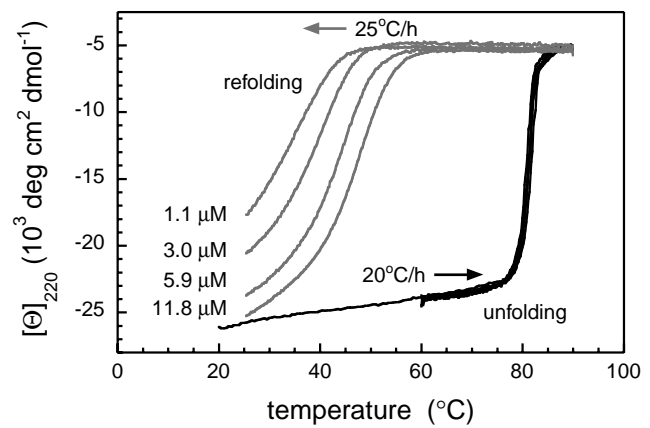

b

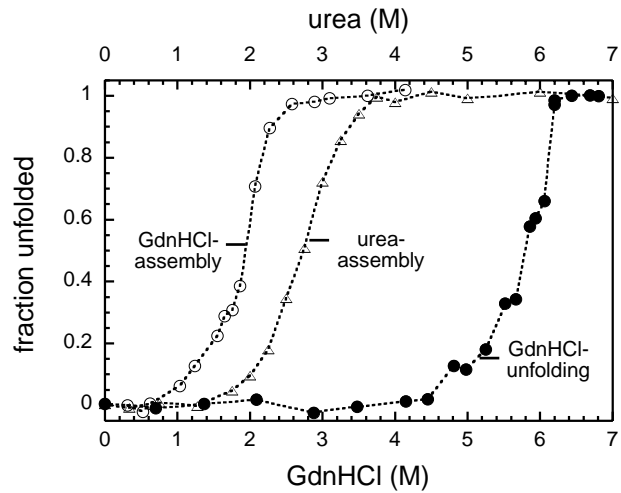

Fig. 1 Unfolding and refolding transitions of the synaptic SNARE complex exhibited a marked hysteresis. a, Thermal unfolding and reconstitution of the synaptic SNARE complex at different protein concentrations monitored by CD spectroscopy at $220 \mathrm{~nm}$. Irreversible unfolding occurred in a steep cooperative transition with $\mathrm{T}_{\mathrm{m}}=82^{\circ} \mathrm{C}\left(20^{\circ} \mathrm{C} \mathrm{h}^{-1}\right)$ (black lines). At a rate of $-5^{\circ} \mathrm{C} \mathrm{h}^{-1}$, slow refolding was observable at $\sim 63^{\circ} \mathrm{C}$ (data not shown). To monitor refolding at different protein concentrations (gray lines), the temperature was lowered from 70 to $25^{\circ} \mathrm{C}$ at $-25^{\circ} \mathrm{C} \mathrm{h}^{-1}$. b, Unfolding and refolding transitions of the synaptic SNARE complex were noncoincident in the presence of denaturants, as monitored by CD spectroscopy at $220 \mathrm{~nm}$. Synaptobrevin, syntaxin and SNAP-25 ( 15 $\mu \mathrm{M}$ of each) were mixed into a GdnHCl- or urea-containing buffer. The ellipticity at $220 \mathrm{~nm}$ was plotted against the denaturant concentration: unfolding in the presence of $\mathrm{GdnHCl}$ (filled circle), refolding in the presence of $\mathrm{GdnHCl}$ (open circle) and refolding in the presence of urea (open triangle).

1Department of Neurobiology and 2Department of Molecular Biology, Max-Planck-Institute for Biophysical Chemistry, Am Fassberg 11, 37077 Göttingen, Germany. 
a

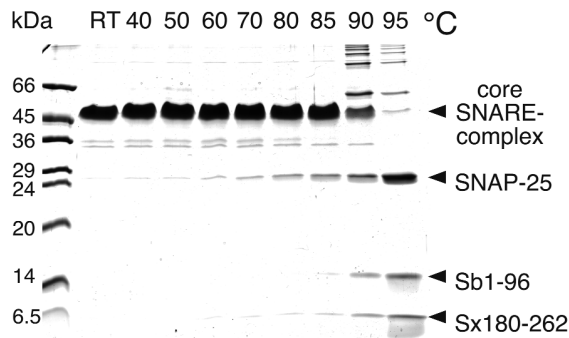

b

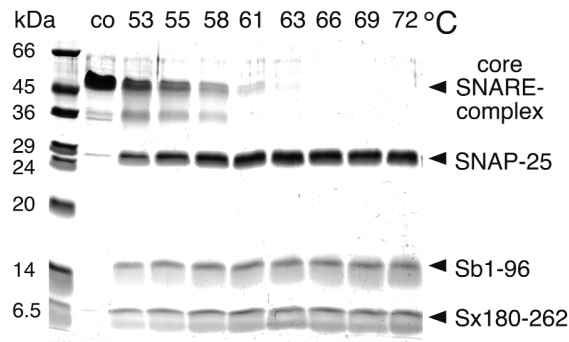

C

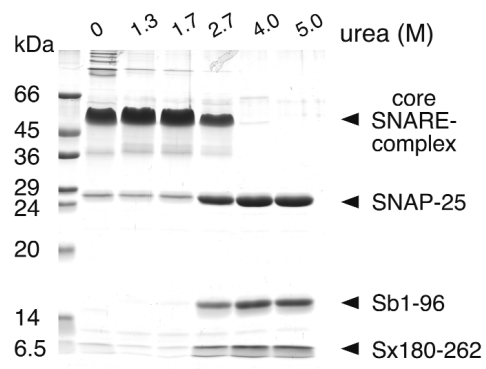

d

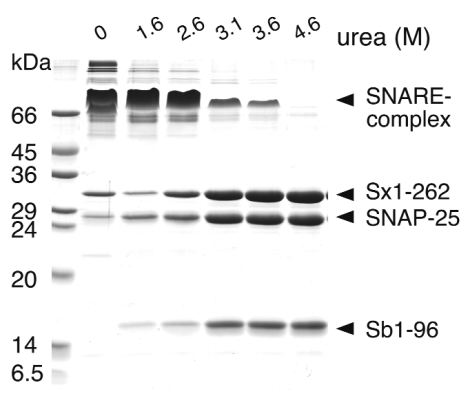

Fig. 2 Hysteresis in the unfolding and folding transition of the synaptic SNARE complex visualized by SDS-PAGE. The assembly status of the SNARES was analyzed by SDS-PAGE and Coomassie blue staining. The assembled complex migrates as a single SDS-resistant band ${ }^{26}$. a, The synaptic complex was incubated at the indicated temperatures for $10 \mathrm{~min}$ and then immediately transferred into SDS sample buffer. The SDS-resistant complex disappeared above $\sim 85^{\circ} \mathrm{C}$, leaving the individual subunits visible. b. Reassembly of the synaptic SNARE complex after unfolding (10 min at $95{ }^{\circ} \mathrm{C}$ ). Before analysis, the samples were incubated at the indicated temperatures for $11 \mathrm{~h}$. Re-formation of the SNARE complex was observable only at temperatures below $\sim 60^{\circ} \mathrm{C}$. An additional band $(\sim 35 \mathrm{kDa})$ is visible upon reconstitution, probably due to some heat-induced protein breakdown because it was not present when the refolding cycle was carried out in urea at room temperature. Assembly of the core synaptic SNARE complex containing c, the SNARE motif of syntaxin (180-262) or d, the full-length cytoplasmic domain of syntaxin (1-262) in the presence of urea. Even at saturating concentration of urea, no unfolding was observed at room temperature (data not shown), whereas SDS-resistant complexes formed only in $\sim 4 \mathrm{M}$ urea. The individual SNARE proteins were mixed in $5 \mathrm{M}$ urea and then diluted into the indicated urea concentrations. After $\sim 1$ week of incubation, the samples were analyzed by SDS-PAGE. The hysteresis in the unfolding and folding transitions was observable regardless of whether the N-terminal domain of syntaxin was present. Co is the control (assembled complex); Sx1-262, syntaxin 1a residues 1-262; Sx180-262, syntaxin 1a residues 180-262; and Sb1-96, synaptobrevin 2 residues 1-96.

Therefore, considerable effort has been made to study SNAREs by a variety of structural approaches ${ }^{11,12}$, mostly using as paradigms the SNARE proteins involved in synaptic exocytosis, including the synaptic vesicle protein synaptobrevin 2 (also referred to as VAM P 2) and the plasma membrane proteins syntaxin $1 A$ and SNAP-25. In solution, the individual SNARE motifs are largely unstructured ${ }^{13-18}$, whereas the crystal structure of the core synaptic SNARE complex shows a tightly packed parallel four-helix bundle, with syntaxin and synaptobrevin each contributing one helix and SNAP-25 contributing two helices ${ }^{19,20}$. Thus, during assembly, SNAREs undergo major conformational rearrangements ${ }^{14}$.

Despite limited sequence homology, the biophysical features of other SNARE proteins, including those involved in Golgi to plasma membrane transport in yeast ${ }^{16,21-23}$ and in the fusion of late endosomes ${ }^{24}$, were shown to be conserved. In contrast to the synaptic and yeast exocytotic complexes, the endosomal SNARE complex, like other SNARE complexes involved in fusion events inside the cell, consists of four rather than three SNARE proteins, each contributing a single $\alpha$-heli $x^{24}$.

A 'binary' complex consisting of one molecule of SNAP-25 and two molecules of syntaxin $1 \mathrm{~A}$ has been suggested to repre sent an intermediate for the assembly of the synaptic SNARE complex ${ }^{14}$. Electron paramagnetic resonance spectroscopy revealed that this complex is also a four-helix bundle, in which a second syntaxin occupies the position of synaptobrevin in the ternary complex 18,25 . When synaptobrevin is added, it displaces the second syntaxin, forming the synaptic SNARE complex ${ }^{14}$.
Although assembled SNARE complexes are very stable ${ }^{14,21,24,26}$, whether this stability translates into sufficient free energy to drive fusion is unclear. This study was initiated to investigate the energetics of SNARE assembly. Because individual SNARE motifs are largely unstructured, assembly can be described as a folding reaction, using reversible denaturation protocols for its characterization. We observed a surprising marked hysteresis in the folding and refolding transition of SNARE complexes, indicating that the free energy landscape is rugged, making the determination of thermodynamic parameters challenging. The syntaxin-SN AP- 25 complex exhibits a reversible folding transition at conditions similar to those for the folding reaction of the synaptic SNARE complex, supporting the idea that this entity might serve as a folding intermediate for the latter.

Un- and refolding hysteresis of synaptic SNARE complex Upon heating, the central four-helix bundle of the synaptic SNARE complex unfolded in a steep cooperative transition ${ }^{15}$ (Fig. 1a). Remarkably, theunfolding transition was not reversible. Refolding was observable only at $\sim 63^{\circ} \mathrm{C}-$ that is, $20^{\circ} \mathrm{C}$ lower than the $T_{m}$ of the unfolding transition (Fig. 1a). No significant changes in the unfolding temperature were observed when different protein concentrations were used (Fig. 1a), substantiating the view that the unfolding transition is not in equilibrium with refolding. Refolding, however, was concentration dependent, as expected for the assembly of independent subunits (Fig. 1a).

Similarly, we observed a hysteresis in the unfolding and refolding transitions in the presence of denaturants. Urea was 
Fig. 3 Unfolding and refolding transitions of an endosomal SNARE complex are noncoincident. Folding and unfolding was monitored by $C D$ spectroscopy at $220 \mathrm{~nm}$. To prevent precipitation, $100 \mathrm{mM} \mathrm{NaCl}$ was present. a, Unfolding and refolding transitions during a heating-cooling cycle. The complex was unfolded with a temperature gradient between 5 and $90{ }^{\circ} \mathrm{C}\left(30{ }^{\circ} \mathrm{C} \mathrm{h}-1\right)$. Refolding was monitored between 90 to $5^{\circ} \mathrm{C}$ $\left(-30{ }^{\circ} \mathrm{C} \mathrm{h}^{-1}\right)$. b. GdnHCl-dependent unfolding and refolding transitions were noncoincident. For unfolding (filled diamond), the complex was incubated in the indicated concentrations of $\mathrm{GdnHCl}$. For reconstitution (open circle, filled circle), the complex was unfolded in $3.5 \mathrm{M} \mathrm{GdnHCl}$ and then renatured in different concentrations of $\mathrm{GdnHCl}(6 \mu \mathrm{M}$ complex). Assembly rates are exceedingly slow (closed symbols are three days of incubation, and open symbols are eleven days of incubation). Addition of $1 \mathrm{M} \mathrm{NaCl}$ to the refolding buffer enhanced the refolding process (squares).

not able to denature the synaptic SNARE complex even when near-saturating concentrations were used ( $9 \mathrm{M}$, data not shown), but unfolding was observed in guanidine hydrochloride ( $\mathrm{GdnHCl}$ ) above $5 \mathrm{M}$ (Fig. 1b). Refolding was monitored upon incubating the three subunits in lower concentrations of $\mathrm{GdnHCl}$ or urea. An increase of $\alpha$-helical content was observed only at $\mathrm{GdnHCl}$ concentrations < 2.5 M (Fig. 1b). No significant change was observed when the samples were measured again after one week, demonstrating that the hysteresis persisted. In urea, complexes formed only below $4 \mathrm{M}$ (Fig. 1b).

To confirm the assembly status of SNARE complexes by a different approach, we separated complexes from monomers by SDS-PAGE. The synaptic SNARE complex is stable in SDS sample buffer, unless heated, and migrates as a single, nondissociated band ${ }^{26}$. Because SDS prevents complex formation, it preserves the ratio between assembled complexes and the uncomplexed subunits. Thus, the effect of SDS depends on the conformational state of the SNARE proteins. To follow unfolding, we incubated the complex at different temperatures and then added SDS sample buffer. At temperatures up to $\sim 85^{\circ} \mathrm{C}$, the complex migrated as a single SDS-resistant band, whereas at higher temperatures it dissociated into its constituents (Fig. 2a).

For refolding, the synaptic SNARE complex was first unfolded by heating to $95^{\circ} \mathrm{C}$. Afterwards each sample was incubated at lower temperatures for $11 \mathrm{~h}$ before SDS sample buffer was added. An SDS-resistant band, however, was observed only at temperatures below $\sim 60^{\circ} \mathrm{C}$ (Fig. 2b). SDSPAGE also confirmed the folding-unfolding hysteresis in the presence of urea. Synaptic SNARE complexes remained intact at up to $9 \mathrm{M}$ urea (data not shown). H owever, when the individual subunits were incubated in decreasing urea concentrations, SDS-resistant complexes became visible only at concentrations below $\sim 4 \mathrm{M}$ urea (Fig. 2c). A very similar hysteresis was observed when the entire cytoplasmic domain, instead of the SNARE motif, of syntaxin was used (Fig. 2d), suggesting that the hysteresis is not influenced by the presence of $\mathrm{N}$-terminal domain of syntaxin.

\section{Similar hysteresis of an endosomal SNARE complex}

To examine whether the hysteresis in the folding-unfolding transition is a general feature of SNARE complexes, we investigated a second complex that functions in endosomal membrane fusion, consisting of the four SNARE proteins endobrevin, syntaxin 7, syntaxin 8 and vtilb that each provide one helix to the four-helix bundle ${ }^{24}$. The complex irreversibly unfolded at $\mathrm{T}_{\mathrm{m}} \sim 78^{\circ} \mathrm{C}$ (Fig. 3a; ref. 24). When the temperature was lowered, refolding was first detectable at $\sim 20^{\circ} \mathrm{C}$. Further cooling to $5^{\circ} \mathrm{C}$ allowed for reformation of $\sim 20 \%$ of the original $\alpha$-helical content (Fig. 3a), which recovered to $\sim 60 \%$ of the original value a

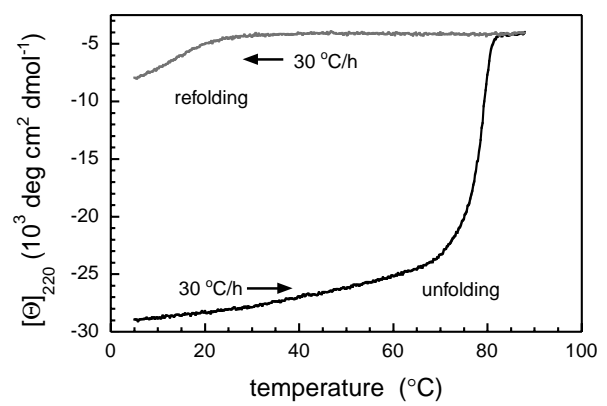

b

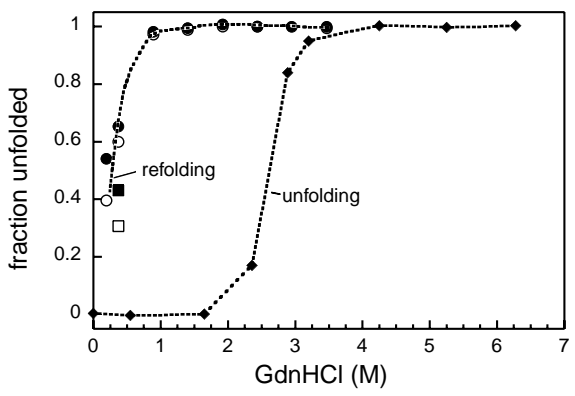

after $2 \mathrm{~d}$ (data not shown). Hence, refolding of the endosomal complex appears to be slower than that of the synaptic SN ARE complex.

In the synaptic complex, two of the helices are linked (in SNAP-25), whereas the two helices in the endosomal complex are represented by two individual proteins, vtilb and syntaxin 8 . To exclude that this difference is responsible for the discrepancy, we examined refolding of a synaptic complex that contained the SNARE motifs of SNAP-25 as separate protein fragments. No major change in the refolding rates was observed (data not shown).

Similarly, a clear hysteresis was observed when unfolding and refolding of the endosomal SNARE complex wereinvestigated in $\mathrm{GdnHCl}$. The complex unfolded at $\sim 2.5 \mathrm{M} \mathrm{GdnHCl}$ (Fig. 3b). Refolding, however, was observable only in $<1 \mathrm{M} \mathrm{GdnHCl}$. Again, the refolding process appeared to be slower than the one observed for the synaptic SNARE complex because a small additional increase in $\alpha$-helical content was observed at permissible $\mathrm{GdnHCl}$ concentrations even after three weeks (data not shown). The presence of a hysteresis implies that assembly follows a different path than dissociation. The assembly reaction was sensitive to temperature - for example, hardly any SNARE complex formation was observed at $25^{\circ} \mathrm{C}$ (Fig. 3a; data not shown). Hence, the intermediate is likely to be unstable; so far, we have been unable to characterize this intermediate in more detail.

\section{Equilibrium unfolding of syntaxin-SNAP-25 complex}

The syntaxin-SNAP-25 complex, which consists of a four-helix bundle structure similar to the synaptic SNARE complex ${ }^{14,18,25}$, reversibly unfolded at $\sim 45^{\circ} \mathrm{C}$ (Fig. $4 \mathrm{a}$ ). The $\mathrm{T}_{\mathrm{m}}$ was el evated with increasing protein concentration, because the syntaxinSNAP-25 complex is formed by independent subunits. An increase in the $\mathrm{NaCl}$ concentration led to stabilization of the syntaxin-SNAP-25 complex $\left(\mathrm{T}_{\mathrm{m}}=57.7^{\circ} \mathrm{C}\right.$ in $\left.1 \mathrm{M} \mathrm{NaCl}\right)$ (Fig. 4a). At all temperatures, the population-weighted superposition of the characteristic spectra for $\alpha$-helix and randomcoil conformations represented the measured spectra well. This was further substantiated by the presence of a well-defined 
a

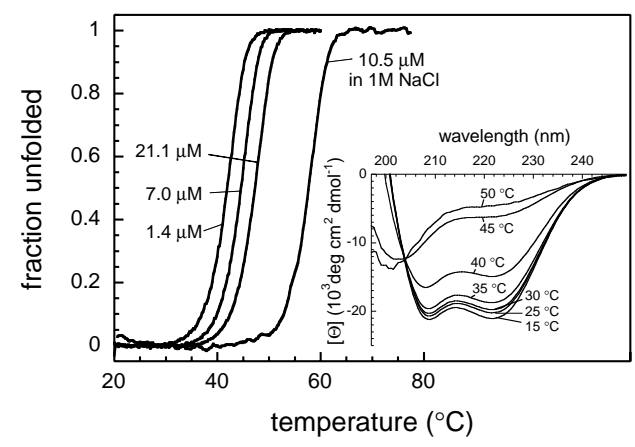

b

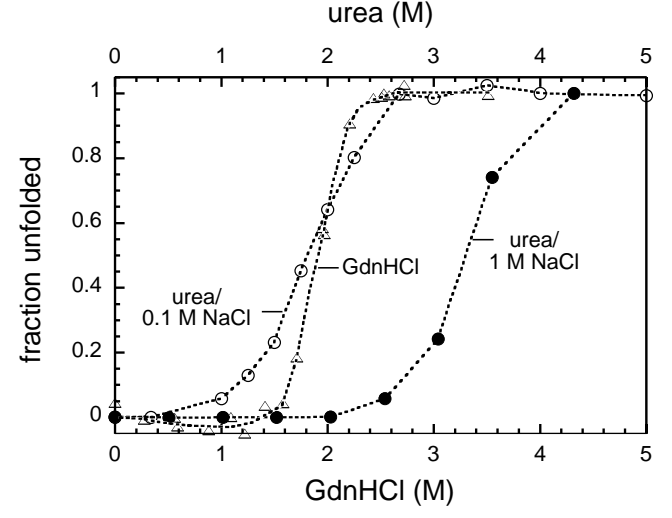

isodichroic point at $204 \mathrm{~nm}$ (Fig. 4a, inset). Hence, the thermal unfolding curve of the syntaxin-SNAP-25 complex suggests a cooperative unfolding process without any detectable intermediate state. The syntaxin-SNAP-25 complex unfolded at $\sim 2 \mathrm{M}$ $\mathrm{GdnHCl}$ (Fig. 4b), similar to the folding transition of the synaptic SNARE complex. In urea, unfolding of the syntaxinSNAP-25 complex required lower concentrations of denaturant than the folding of the synaptic SNARE complex (Figs 1b, 4b), although the unfolding of the syntaxin-SNAP-25 complex in urea was dependent on theionic strength.

\section{Unfolding kinetics}

The rate of unfolding of the synaptic SNARE complex was determined by monitoring the disappearance of $\alpha$-helical content following dilution into a series of $\mathrm{GdnHCl}$ concentrations. Over the range of 5.8-6.8 $\mathrm{M}$, the denaturation time course was amenable to evaluation by manual mixing methods. To determine the rate of unfolding at each concentration of $\mathrm{GdnHCl}$, each reaction was fit to a single exponential (Fig. 5a, inset). The kinetics of unfolding showed a linear dependence of In $\mathrm{k}$ on the concentration of denaturant. Hence, the data can be extrapolated to native conditions (zero denaturant) to an estimated first order rate constant of dissociation of the synaptic SNARE complex of $\sim 4.2 \times 10^{-18} \mathrm{~s}^{-1}$ (Fig. 5a). This rate is so slow that the half-life of the complex would be more than a billion years, suggesting that once SNARE complex forms, it does not dissociate on a biologically relevant time scale under nondenaturing conditions. However, this interpretation assumes that the ratedetermining step does not change under physiological conditions. Similarly, the rate of unfolding for the syntaxin-SNAP-25 complex was determined (Fig. 5b, inset). When extrapolated to native conditions, the first order rate constant of dissociation was $\sim 5.2 \times 10^{-7} \mathrm{~s}^{-1}$ (Fig. 5b), which corresponds to a half-life of $\sim 11 \mathrm{~d}$.
Fig. 4 Equilibrium unfolding of the syntaxin-SNAP-25 complex monitored by $C D$ spectroscopy. a, Reversible thermal unfolding of the syntaxin -SNAP-25 complex. The $\mathrm{T}_{m}$ of the unfolding transition increased with increasing protein concentration (at $1.4 \mu \mathrm{M}, \mathrm{T}_{\mathrm{m}}=42{ }^{\circ} \mathrm{C}$; at $7 \mu \mathrm{M}$, $\mathrm{T}_{\mathrm{m}}=44.5^{\circ} \mathrm{C}$; and at $21.1 \mu \mathrm{M}, \mathrm{T}_{\mathrm{m}}=47.3^{\circ} \mathrm{C}$ ). In the presence of $1 \mathrm{M} \mathrm{NaCl}$, a shift in the unfolding transition to higher temperatures was observed (at $10.5 \mu \mathrm{M}, \mathrm{T}_{\mathrm{m}}=57.7^{\circ} \mathrm{C}$ ). Inset, The far UV CD-spectra of the syntaxin -SNAP-25 complex $(7.0 \mu \mathrm{M})$ recorded at different temperatures exhibited an isodichroic point at $204 \mathrm{~nm}$, suggesting that only $\alpha$-helical and random coiled conformations were present at each temperature. $\mathbf{b}$, Unfolding of the syntaxin-SNAP-25 complex in the presence of denaturants. In urea and $1 \mathrm{M} \mathrm{NaCl}$, the complex unfolded at a half-point of $\sim 3.25 \mathrm{M}$ urea $(7 \mu \mathrm{M}$ complex, filled circle), whereas in $100 \mathrm{mM} \mathrm{NaCl}$, unfolding occurred at $\sim 1.8 \mathrm{M}$ urea (15 $\mu \mathrm{M}$ mixed subunits, open circle). In $\mathrm{GdnHCl}$, the complex unfolded at a half-point of $\sim 1.8 \mathrm{M} \mathrm{GdnHCl}(7 \mu \mathrm{M}$, open triangle).

\section{Folding kinetics}

To assess the refolding rates, complexes were completely unfolded in $\mathrm{GdnHCl}$ and subsequently refolded by dilution. Remarkably, the overall slow refolding process of the synaptic SNARE complex in the presence of $\mathrm{GdnHCl}$ exhibited two clearly visible phases: a fast first phase with a half life of $\sim 1$ min, followed by a slow second component with a half life of $\sim 1 \mathrm{~h}$ (at $0.42 \mathrm{M} \mathrm{GdnHCl}$, Fig. 6a). Refolding of the syntaxin-SNAP-25 complex exhibited only one phase (Fig. 6b). A preliminary analysis suggests that the half life of the fast first phase of the refolding of the synaptic SNARE complex was similar to that of the assembly reaction of the syntaxin-SNAP-25 complex (Fig. 6c). Both exhibited a similar dependence on the $\mathrm{GdnHCl}$ concentration, whereas the second phase of the synaptic SNARE complex refolding seemed not to change substantially with the concentration of $\mathrm{GdnHCl}$. More interesting, the two steps in assembly of the synaptic SNARE complex were clearly evident only in the presence of $\mathrm{GdnHCl}$. Only the fast component was enhanced when syntaxin or SNAP- 25 was added to the refolding of the synaptic SNARE complex, corroborating the similarity of the assembly path of both complexes. In contrast, addition of synaptobrevin had hardly any effect on the first phase (data not shown).

Next, we tested whether the formation of the synaptic SNARE complex is accelerated in the presence of the syntaxin-SNAP-25 complex. When the syntaxin-SNAP-25 complex was mixed with synaptobrevin, the formation of an SDS-resistant complex occurred within $\sim 2$ min (data not shown). In the presence of $2 \mathrm{M}$ urea - that is, a concentration that does not dissociate the syntaxin-SNAP-25 complex - the reaction was slightly retarded, with a half life of $\sim 5-10$ min (Fig. $6 d$ ). The reaction rate was significantly slowed, however, when the syntaxinSNAP-25 complex was first unfolded and then mixed with synaptobrevin in $2 \mathrm{M}$ urea (Fig. 6e); an SDS-resistant complex started to appear only after 5-10 min. A similar rate was observed when the reaction was started with theindividual components in $2 \mathrm{M}$ urea (data not shown). Together, our data suggest that the syntaxin-SNAP-25 complex and the synaptic SNARE complex assemble via similar pathways.

\section{Discussion}

In this study, we employed folding and unfolding paradigms to learn more about the energetics and kinetics of SNARE complex formation. Surprisingly, we found that assembly and dissociation appear to follow different reaction paths, as evident by a pronounced hysteresis in thefolding-unfolding transitions. This hysteresis seems to be a common principle for SNARE complexes because it was seen in two different, distantly related complexes. Moreover, the hysteresis was not influenced by the presence of the autonomous $\mathrm{N}$-terminal domain of syntaxin 
Fig. 5 Unfolding kinetics of the synaptic complex monitored by CD spectroscopy at $220 \mathrm{~nm}$. a, Unfolding kinetics of the synaptic SNARE complex in $\mathrm{GdnHCl}$ (inset, black lines). A $75 \mu \mathrm{l}$ sample $\left(\sim 1 \mathrm{mg} \mathrm{ml}^{-1}\right)$ was mixed into a solution containing $525 \mu \mathrm{l} \mathrm{GdnHCl}$ in a $0.2 \mathrm{~cm}$ cuvette. The unfolding kinetics were fit as a monomolecular reaction (red lines). The logarithm of the first-order rate constants obtained from the fit were plotted against the concentration of $\mathrm{GdnHCl}$. The rate constant for the unfolding reaction, extrapolated to zero denaturant, was $4.2 \times 10^{-18} \mathrm{~s}^{-1}$. b, Unfolding kinetics of the syntaxin-SNAP-25 complex in GdnHCl (inset, black lines). The syntaxin-SNAP-25 complex ( $40 \mu \mathrm{l}$ of $\sim 2 \mathrm{mg} \mathrm{ml}^{-1}$ ) was diluted into $560 \mu \mathrm{GdnHCl}$-containing solution. The unfolding kinetics were fit as a monomolecular reaction (red lines). The logarithm of the first-order rate constants obtained from the fit were plotted against the concentration of $\mathrm{GdnHCl}$. The rate constant for the unfolding reaction, extrapolated to zero denaturant, was $5.2 \times 10^{-7} \mathrm{~s}^{-1}$.

and, thus, represents an intrinsic feature of the core SNARE complex.

A high energetic barrier between the folded and unfolded states causes the observed hysteresis. Consequently, the rates of dissociation and assembly are slow. Thus, in certain conditions the dissociated subunits are able to coexist with assembled complex without any measurable interconversion. Although these properties prevented us from determining an equilibrium constant for SNARE complex formation, our data shed new light on possible intermediates during assembly and, furthermore, have implications for the mechanism of SNAREs in membrane fusion.

M any small globular proteins reside at a global minimum of free energy in their folded state. For these proteins, the native state is in equilibrium with the unfolded state, implying that the kinetic barriers between the folded and unfolded states are readily surmountable 27,28 . However, for more complex proteins, kinetic factors can play a critical role in dictating the final structure. Thesenative structures must have sufficient kinetic stability to exist on a biological time scale, but they do not necessarily have to be at a global minimum of free energy 29,30. A hysteresis in the denaturation and refolding curves that is similar to what we have found for SNARE interaction has been reported for other proteins, including the oligomeric proteins bacterial luciferase and transthyretin ${ }^{31,32}$. Thus far, however, there was no precedent for an association and dissociation reaction of profound biological significance that exhibited such unusual properties. Only the availability of an ATP-driven disassembly mechanism may have permitted the evolution of a kinetically stabilized SNARE interaction.

\section{An intermediate is required for SNARE assembly}

A hysteresis in the folding and refolding of SNARE complexes suggests the existence of a folding intermediate. Interestingly, folding of the synaptic syntaxin-SNAP-25 complex did not show hysteresis. This complex consists of a four-helix bundle similar to the SNARE complex, but a second syntaxin helix occupies the binding site of synaptobrevin 18,25 . The folding equilibrium of the syntaxin-SNAP-25 complex occurred at conditions similar to those permitting assembly of the synaptic SNARE complex. Thus, the SNARE motifs of syntaxin and SNAP-25 might have to interact before synaptobrevin can bind. This view is corroborated by the apparent biphasic assembly of the synaptic SNARE complex in the presence of $\mathrm{GdnHCl}$. The faster phase probably corresponds to the assembly of syntaxin and SNAP-25, followed by a slow further increase of $\alpha$-helical content that may represent the binding of synaptobrevin. Furthermore, the presence of the syntaxin-SNAP-25 complex seemed to accelerate the formation of the synaptic SNARE a

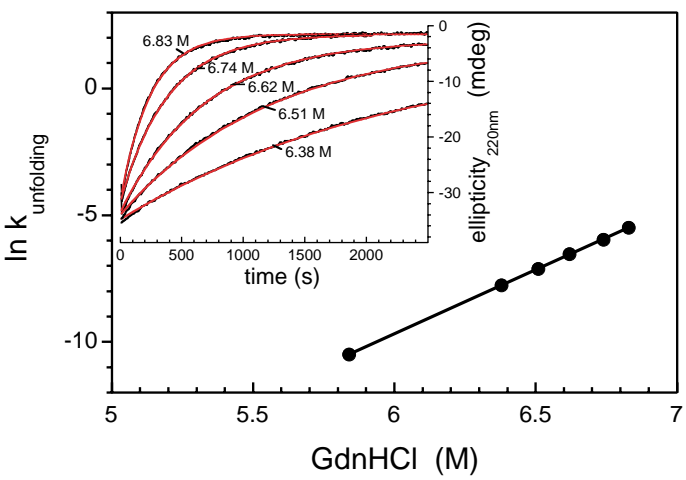

b

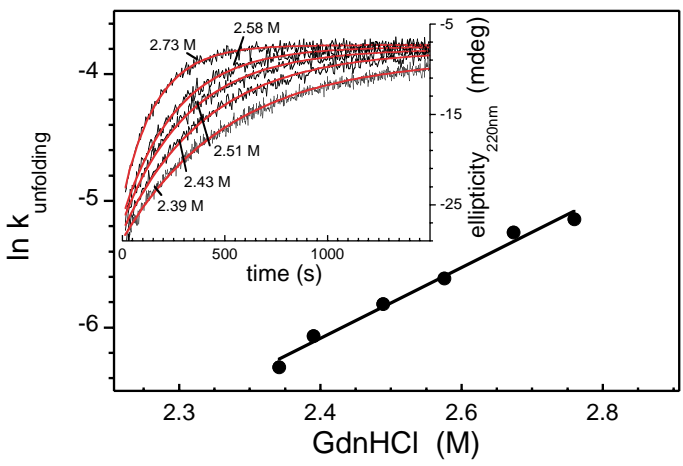

complex. Thus, even when the autonomous $\mathrm{N}$-terminal domain of syntaxin ${ }^{16,22}$ was absent, the syntaxin-SNAP-25 interaction seemed to serve as a nucleation site for the assembly of the synaptic SNARE complex.

The question arises whether the syntaxin-SNAP-25 complex needs to be fully assembled before synaptobrevin binds. Alternatively, a transient intermediate containing only one copy of syntaxin may serve as the 'true' synaptobrevin acceptor. In this context, the corresponding binary complex in yeast contains only a single copy of the syntaxin homolog Sso 1 (refs 16,21,22). Thus, transient complexes consisting of three SNARE motifs one from syntaxin and two from SNAP-25 homologs - may function as the synaptobrevin acceptor in vivo.

The intermediate involved in the formation of the endosomal complex was much less stable than that of the synaptic complex. Therefore, identifying and isolating the intermediate was not possible, preventing us from determining its composition. Preliminary results involving endosomal SNAREs reconstituted in liposomes suggest that the intermediate may be more stable under such conditions of the liposomes (unpublished observations). However, the endosomal R-SNARE endobrevin is able to use the synaptic syntaxin-SNAP-25 complex as an intermediate to form a 'mixed' SNARE complex ${ }^{33}$.

Of all binary combinations, only SNAP-25 and syntaxin form a stablecomplex ${ }^{14}$; however, other stable assemblies form in vitro when the linker region between the two helices of SNAP-25 is removed. These include complexes containing syntaxin, synaptobrevin and either the $\mathrm{N}$ - or the C-terminal helix of SNAP-25, and complexes of syntaxin and the $\mathrm{N}$-terminal helix of SNAP-25 (refs 13,15,34). Whether such complexes play a role during SNARE assembly remains to be elucidated.

Clearly, SNARE-assembly and disassembly are complex, multistep reactions, and more refined structural approaches are required to further elucidate the intermediate steps. Our data support the view that the plasma membrane proteins syntaxin 
a

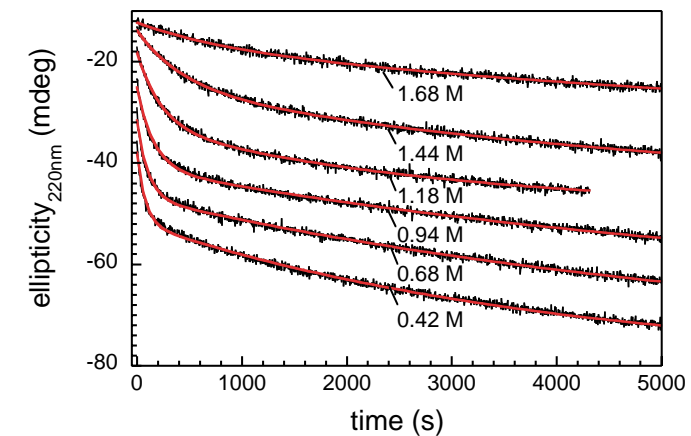

b

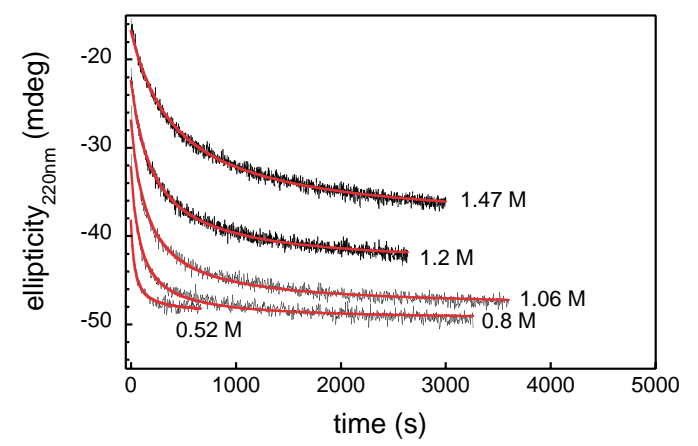

C

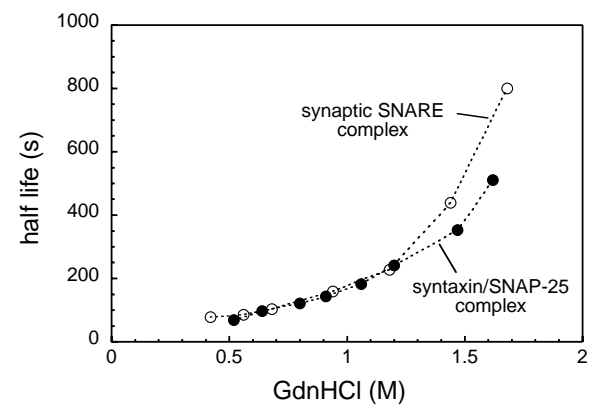

e

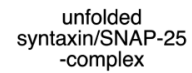

co $1^{\prime} 2^{\prime} 5^{\star} 10^{\star} 15^{`} 30^{\prime} 1 \mathrm{~h} 2 \mathrm{~h} 18 \mathrm{~h}$

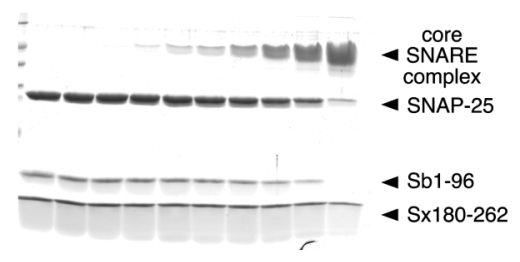

Fig. 6 Biphasic refolding kinetics of the synaptic SNARE complex suggest assembly via a syntaxin-SNAP-25 intermediate. a, Refolding kinetics of the synaptic SNARE complex measured by CD spectroscopy at $220 \mathrm{~nm}$. Unfolded complex $\left(\sim 1 \mathrm{mg} \mathrm{ml}^{-1}\right.$ in $\left.8 \mathrm{M} \mathrm{GdnHCl}\right)$ was diluted into buffer containing different $\mathrm{GdnHCl}$ concentrations (final protein concentration $4.3 \mu \mathrm{M}$ ). Refolding kinetics exhibited two phases (black lines are $7.2 \mu \mathrm{M}$ complex, and red lines are the double exponential fit). After 10 days, all samples, except refolding at $1.68 \mathrm{M} \mathrm{GdnHCl}$, had reached similar $\alpha$-helical content $(\sim 90 \mathrm{mdeg}$ ). At $0.42 \mathrm{M} \mathrm{GdnHCl}$, the contribution of the fast phase (half life of $\sim 78 \mathrm{~s}$ ) to the amplitude was $\sim 50 \%$. The half-life of the slow phase was $\sim 1 \mathrm{~h}$. Above $1.44 \mathrm{M} \mathrm{GdnHCl}$, only the slow phase was detectable. b, Refolding kinetics of the syntaxin-SNAP-25 complex measured by CD spectroscopy at $220 \mathrm{~nm}$. The complex was unfolded in $2.5 \mathrm{M} \mathrm{GdnHCl}$ and diluted (black lines are $7.2 \mu \mathrm{M}$ complex, and red lines are single exponential fit). Refolding kinetics exhibited only one phase. At $0.52 \mathrm{M} \mathrm{GdnHCl}$, the half-life of refolding was $\sim 68 \mathrm{~s}$. c, Comparison of the folding rate constants of the synaptic SNARE complex (rapid phase =open circles) with those of the syntaxin-SNAP-25 complex (closed circles) at increasing GdnHCl concentrations. The refolding rates of the synaptic SNARE complex and the syntaxin-SNAP-25 complex were determined by double and single exponential fits, respectively. d,e, Formation of the synaptic SNARE complex was accelerated when the syntaxin-SNAP-25 complex is preformed. The time-dependence of the formation of the SDS-resistant synaptic SNARE complex is monitored by SDS-PAGE and Coomassie blue staining. To the preformed syntaxin-SNAP-25 complex in (c), synaptobrevin was added (10 $\mu \mathrm{M}$ protein) in the presence of $2 \mathrm{M}$ urea. In (d), the syntaxin-SNAP-25 complex was first unfolded in $5 \mathrm{M}$ urea and diluted shortly before synaptobrevin was added (final concentration $=2 \mathrm{M}$ urea). Co is the control (heat-dissociated complex); other abbreviations are as in Fig. 2.

and SNAP-25 form thein vivo acceptor site for the vesicle protein synaptobrevin. This is corroborated by the finding that liposomes reconstituted with synaptic SNARE proteins appear to fuse only when syntaxin and SNAP-25 were coreconstituted into one population of liposomes?.

\section{Biological relevance of the hysteresis}

The slow assembly rates appear to be hallmarks of all SNARE complexes; however, they are difficult to reconcilewith their biological function. For example, synaptic exocytosis occurs in fractions of a second. The conditions for SNARE assembly, however, are vastly different within cells than in vitro. First, themembrane anchors may lead to high local concentrations and, furthermore, put steric constraints on the assembly reaction. Nevertheless, membrane anchoring al one does not appear to accelerate assembly because SNARE-mediated liposome fusion is slow and occurs at a time-scale comparable to that for the assembly in solution?. Second, SNAREs may not be randomly structured but constrained by interactions with accessory proteins. Ther efore, if SNARE assembly is indeed part of the rapid membrane fusion mechanism, its slow intrinsic rates demand accelerating factors.

But why has such 'sluggish' machinery been utilized? Apart from the probable important energetic contribution of SNARE assembly to fusion, the unusual properties of SNARE allow for a tight spatial and temporal control of the assembly reaction. In addition, a slow assembly rate might provide a kinetic barrier protecting cells against inadvertent fusion reactions.

Likewise, the enormous kinetic stability of the assembled complex may be directly related to SNARE function. Disassembly of the complex requires the ATPase NSF and its cofactors, which are essential and very abundant proteins. If the assembly of trans-SNARE complexes occurs in an ordered, zipper-like fashion, the reaction may become progressively more irreversible, due to a kinetic trap, until a 'point of no return' is reached. That such assembly intermediates are substrates for NSF cannot be excluded, as was suggested by experiments carried out on SNAREs involved in yeast vacuole fusion?. Nevertheless, the virtually irreversible nature of the assembly reaction would be perfectly suited to overcome the repulsive forces between membranes. Assembly into trans-complexes would beableto providea tight connection between membranes and eventually initiate fusion ${ }^{6}$.

\section{Methods}

SNARE complex assembly and purification. All recombinant proteins used in this study have been described $15,24,33$. Proteins and protein complexes were purified by affinity chromatography followed by ion exchange chromatography on a FPLC system (Äkta, Amersham Pharmacia Biotech) essentially as described ${ }^{15,24,33}$. 
The following complexes were assembled and purified. The synaptic SNARE complex composed of the (i) SNARE motif of syntaxin 1A (residues 180-262) or (ii) the full cytoplasmic domain of syntaxin 1A (1-262), SNAP-25 (1-206) and synaptobrevin (residues 1-96); (iii) the syntaxin-SNAP-25 complex composed of two molecules of the syntaxin 1A SNARE-motif (residues 180-262) and one molecule of SNAP-25; and (iv) the endosomal SNARE complex composed of the SNARE motifs of syntaxin 7 (residues 159-236), syntaxin 8 (residues 136-213), vtilb (residues 130-206) and endobrevin (residues 1-74). Protein concentrations were determined by absorption at $280 \mathrm{~nm}$ in $6 \mathrm{M} \mathrm{GdnHCl}$ and/or using the Bradford assay.

Circular dichroism (CD) spectroscopy. CD spectroscopy was carried out essentially as described ${ }^{24,33}$. If not stated otherwise, all heat and $\mathrm{GdnHCl}$ denaturations were carried out in $40 \mathrm{mM}$ sodium phos phate, $\mathrm{pH} 7.4$, without $\mathrm{NaCl}$. For all urea denaturations, $20 \mathrm{mM}$ Tris-HCl, pH 7.4, was used. For thermal denaturation and refolding experiments, the ellipticity at $220 \mathrm{~nm}$ was recorded as a function of temperature, as indicated in the figure legends. In order to reduce heat-induced protein damage during refolding of the synaptic complex, the temperature was lowered rapidly from $90^{\circ} \mathrm{C}$ to $70^{\circ} \mathrm{C}$ $\left(-60{ }^{\circ} \mathrm{C} \mathrm{h}^{-1}\right)$. To record the folding transitions at different protein concentrations, cuvettes with different path lengths were used. For the synaptic SNARE complex, cooling to $25^{\circ} \mathrm{C}$ after heat denaturation resulted in the formation of $\sim 80-95 \%$ of the original $\alpha$-helical content. Hence, few off-pathway reactions occur upon refolding. A similar folding transition at $82{ }^{\circ} \mathrm{C}$ was measured when a heatunfolded sample was subjected to a second heat-denaturation after removal of small amounts of aggregates. Likewise, when an unfolded sample was reheated, followed by cooling until $~ 50 \%$ of its original $\alpha$-helical structure was reformed, a similar unfolding transition occurred at $82{ }^{\circ} \mathrm{C}$. This indicates that a similar complex had been formed upon refolding. Unfolding and refolding experiments using denaturants were carried out at $25^{\circ} \mathrm{C}$. Urea and $\mathrm{GdnHCl}$ stock solutions were prepared gravimetrically and controlled by refractive indices. Routinely, the denaturant concentration of each solution was determined again after data collection.

For equilibrium studies, each CD spectrum was recorded after incubation for at least $24 \mathrm{~h}$. For equilibrium unfolding, complexes were subjected to different concentrations of urea or $\mathrm{GdnHCl}$. For unfolding kinetics, complexes were mixed rapidly into $\mathrm{GdnHCl}$-containing buffer, and the change in the $C D$ signal was followed at $220 \mathrm{~nm}$. The manual mixing process took $\sim 20 \mathrm{~s}$. Equilibrium refold- ing was measured in different denaturant concentrations. Therefore, complexes were first unfolded in denaturant concentrations sufficient for complete unfolding and then diluted. For refolding kinetics of unfolded complexes, the ellipticity at $220 \mathrm{~nm}$ was recorded as a function of time. The first data points were taken $<10$ s after mixing. We noted that the CD approach is limited for assembly kinetics, because it indicates only the total increase in helicity without shedding light on the conformational changes of individual subunits.

Data analysis. Raw data from equilibrium unfolding experiments were analyzed according to standard procedures ${ }^{35}$. For unfolding kinetics, the first-order rate constant was evaluated at a number of different $\mathrm{GdnHCl}$ concentrations. The change of signal could be fit to the equation $Y=Y_{A}[A]+Y_{B}\left(\left[A_{0}\right]-[A]\right)$, where $Y=$ $C D$ signal, $Y_{A}=$ signal of complex, $Y_{B}=$ signal of unfolded monomers, $\left[A_{0}\right]=$ initial complex concentration and $[A]=$ the concentration of complex determined by the first-order rate equation $[\mathrm{A}]=[\mathrm{A}]_{0} \mathrm{e}^{-\mathrm{kt}}$, where $\mathrm{k}=$ first-order rate constant and $\mathrm{t}=$ time in seconds. Refolding data were analyzed using either a single or a double exponential fit. Kaleidagraph (Synergy Software) was used to fit the data.

Electrophoretic procedures. When testing SDS resistance, samples were treated at the conditions indicated in the figure panels and transferred into SDS sample buffer (final concentrations are $60 \mathrm{mM}$ Tris, pH 6.8, 2\% (w/v) SDS, 10\% (w/v) glycerol and 3\% (v/v) $\beta$-mercaptoethanol) before analysis on a $15 \%$ polyacrylamide gel26,33. For reconstitution of the SDS-resistant synaptic SNARE complex, the complex was unfolded for $10 \mathrm{~min}$ at $95^{\circ} \mathrm{C}$ and then incubated at lower temperatures for $11 \mathrm{~h}$ in a Mastercycler gradient (Eppendorf).

\section{Acknow ledgments}

The authors are very much indebted to $\mathrm{K}$. Fiebig for pointing out in the beginning of the study that the observed hysteresis could be due to a folding intermediate. We thank M. Margittai, R. Langen, D. Bruns, T. Heimburg, S. Pabst, V. Knecht, G. Schröder and H. Grubmüller for stimulating discussions, suggestions and critical reading of the manuscript.

Received 18 September, 2001; accepted 27 November, 2001. 
1. Palade, G. Intracellular aspects of the process of protein synthesis. Science $\mathbf{1 8 9}$ 347-358 (1975).

2. Zimmerberg, J. \& Chernomordik, L.V. Membrane fusion. Adv. Drug. Deliv. Rev. 38, 197-205 (1999)

3. Lentz, B.R., Malinin, V., Haque, M.E. \& Evans, K. Protein machines and lipid assemblies: current views of cell membrane fusion. Curr. Opin. Struct. Biol. 10 607-615 (2000).

4. Jahn, R. \& Südhof, T.C. Membrane fusion and exocytosis. Annu. Rev. Biochem. 68 863-911 (1999).

5. Lin, R.C. \& Scheller, R.H. Mechanisms of synaptic vesicle exocytosis. Annu. Rev. Cell Dev. Biol. 16, 19-49 (2000)

6. Hanson, P.I., Heuser, J.E. \& Jahn, R. Neurotransmitter release - four years of SNARE complexes. Curr. Opin. Neurobiol. 7, 310-315 (1997).

7. Weber, T. et al SNAREpins: minimal machinery for membrane fusion. Cell 92 759-772 (1998)

8. Ungermann, C., Sato, K. \& Wickner, W. Defining the functions of trans-SNARE pairs. Nature 396, 543-548 (1998).

9. Peters, C. \& Mayer, A. Ca2+/calmodulin signals the completion of docking and triggers a late step of vacuole fusion. Nature 396, 575-580 (1998).

10. Peters, C. et al. Trans-complex formation by proteolipid channels in the termina phase of membrane fusion. Nature 409, 581-588 (2001).

11. Misura, K.M., May, A.P. \& Weiss, W.I. Protein-protein interactions in intracellular membrane fusion. Curr. Opin. Struct. Biol. 10, 662-671 (2000).

12. Brünger, A.T. Structural insights into the molecular mechanism of calcium dependent vesicle-membrane fusion. Curr. Opin. Struct. Biol. 11, 163-73 (2001).

13. Fasshauer, D., Bruns, D., Shen, B., Jahn, R. \& Brünger, A.T. A structural change occurs upon binding of syntaxin to SNAP-25. J. Biol. Chem. 7, 4582-4590 (1997)

14. Fasshauer, D., Otto, H., Eliason, W.K., Jahn, R. \& Brünger, A.T. Structural changes are associated with soluble $\mathrm{N}$-ethylmaleimide-sensitive fusion protein attachment protein receptor complex formation. J. Biol. Chem. 272. 28036-28041 (1997).

15. Fasshauer, D., Eliason, W.K., Brünger, A.T. \& Jahn, R. Identification of a minima core of the synaptic SNARE complex sufficient for reversible assembly and disassembly. Biochemistry 37, 10354-10362 (1998)

16. Fiebig, K.M., Rice, L.M., Pollock, E. \& Brünger, A.T. Folding intermediates of SNARE complex assembly. Nature Struct. Biol. 6, 117-123 (1999).

17. Hazzard, J., Sudhof, T.C. \& Rizo, J. NMR analysis of the structure of synaptobrevin and of its interaction with syntaxin. J. Biomol. NMR 14, 203-207 (1999).

18. Margittai, M., Fasshauer, D., Pabst, S., Jahn, R. \& Langen, R. Homo- and heterooligomeric snare complexes studied by site-directed spin labeling. J. Biol. Chem. 276, 13169-77. (2001).
19. Sutton, R.B., Fasshauer, D., Jahn, R. \& Brünger, A.T. Crystal structure of a SNARE complex involved in synaptic exocytosis at $2.4 \AA$ resolution. Nature $395,347-53$ (1998).

20. Fasshauer, D., Sutton, R.B., Brünger, A.T. \& Jahn, R. Conserved structural features of the synaptic fusion complex: SNARE proteins reclassified as Q- and R-SNAREs. Proc. Natl. Acad. Sci. USA 95, 15781-15786 (1998).

21. Rice, L.M. Brennwald, P. \& Brünger, A.T. Formation of a yeast SNARE complex is accompanied by significant structural changes. FEBS Lett. 415, 49-55 (1997).

22. Nicholson, K.L. et al. Regulation of SNARE complex assembly by an N-terminal domain of the t-SNARE Ssolp. Nature Struct. Biol. 5, 793-802 (1998).

23. Munson, M., Chen, X., Cocina, A.E., Schultz, S.M. \& Hughson, F.M. Interactions within the yeast t-SNARE ssolp that control SNARE complex assembly. Nature Struct. Biol. 7, 894-902 (2000).

24. Antonin, W. et al. A SNARE complex mediating fusion of late endosomes defines conserved properties of SNARE structure and function. EMBO J. 19, 6453-6464 (2000).

25. Xiao, W Poirier M.A. Bennett, M.K. \& Shin, Y.K. The neuronal t-SNARE complex is a parallel four-helix bundle. Nature Struct. Biol. 8, 308-311 (2001).

26. Hayashi, T. et al. Synaptic vesicle membrane fusion complex: action of clostridial neurotoxins on assembly. EMBO J. 13, 5051-5061 (1994).

27. Anfinsen, C.B. Principles that govern the folding of protein chains. Science 181, 223-230 (1973).

28. Dill, K.A. Folding proteins: finding a needle in a haystack. Curr. Opin. Struct. Biol. 3, 99-103 (1993)

29. Baker, D. \& Agard, D.A. Kinetics versus thermodynamics in protein folding. Biochemistry 33, 7505-7509 (1994).

30. Plaza del Pino, I.M., Ibarra-Molero, B. \& Sanchez-Ruiz, J.M. Lower kinetic limit to protein thermal stability: a proposal regarding protein stability in vivo and its relation with misfolding diseases. Proteins 40, 58-70 (2000).

31. Sinclair, J.F., Ziegler, M.M. \& Baldwin, T.O. Kinetic partitioning during protein folding yields multiple native states. Nature Struct. Biol. 1, 320-326 (1994).

32. Lai, Z., McCulloch, J., Lashuel, H.A. \& Kelly, J.W. Guanidine hydrochlorideinduced denaturation and refolding of transthyretin exhibits a marked hysteresis: equilibria with high kinetic barriers. Biochemistry 36, 10230-10239 hysteresis:

33. Fasshauer, D., Antonin, W., Margittai, M., Pabst, S. \& Jahn, R. Mixed and noncognate SNARE complexes. Characterization of assembly and biophysical properties. J. Biol. Chem. 274, 15440-15446 (1999).

34. Poirier, M.A. et al. Protease resistance of syntaxin.SNAP-25.VAMP complexes. Implications for assembly and structure. J. Biol. Chem. 273, 11370-11377 (1998).

35. Pace, C.N. The stability of globular proteins. CRC Crit. Rev. Biochem. 3, 1-43. (1975). 